\title{
Relationship between Thermal Stability and Structure of MEMS Cantilevers Embedded in an Elastomer
}

\author{
Toshihiro Takeshita, ${ }^{1 *}$ Natsumi Makimoto, ${ }^{1}$ \\ Takeshi Kobayashi, ${ }^{1}$ and Seiichi Takamatsu ${ }^{2}$ \\ ${ }^{1}$ National Institute of Advanced Industrial Science and Technology (AIST), \\ 1-2-1, Namiki, Tsukuba, Ibaraki 305-0028, Japan \\ ${ }^{2}$ The University of Tokyo, 5-1-5, Kashiwanoha, Kashiwa, Chiba 277-8561, Japan
}

(Received April 30, 2020; accepted July 22, 2020)

Keywords: MEMS, force sensor, cantilever, thermal endurance, PDMS

The thermal stability of a micro-electromechanical system (MEMS) cantilever force sensor embedded in an elastomer was examined. When mounting the cantilever on an electronic substrate using solder reflow at $250{ }^{\circ} \mathrm{C}$, the elastomer expands against the back of the cantilever. The expansion often breaks the cantilever. The effects of the cantilever thickness and width on the breaking strength and the stress concentration points were simulated by finite element methods, and the results agreed well with experimental results. This work is expected to improve the thermal stability of MEMS cantilever force sensors by optimizing their design.

\section{Introduction}

In robotics, the demand for small devices such as displacement sensors, ${ }^{(1,2)}$ micro-rotary encoders, ${ }^{(3,4)}$ torque sensors, ${ }^{(5,6)}$ and force sensors ${ }^{(7-14)}$ has been increasing. In particular, for robots that must grasp objects, such as industrial and medical robots, a small force sensor is very important. This is because the objects grasped by the robotic hand can vary from hard objects such as machines and electrical components to soft objects such as food and tissue. For soft objects, the grasp must be controlled with optimal forces that prevent both destruction and slippage. Therefore, high accuracy, high resolution, and a small size are characteristics required of the force sensor.

A force sensor fabricated from a micro-electromechanical system (MEMS) cantilever and then embedded in an elastomer is expected to meet these requirements. Shimoyama and coworkers reported on a MEMS cantilever that enabled simultaneous measurements of pressure and shear forces. It was oriented vertically by a magnetic field and was embedded in poly(dimethylpolysiloxane) (PDMS). ${ }^{(10,11)}$ Pressure force measurements were performed in three directions, while shear forces were measured along two axes. Sohgawa and coworkers also developed a force sensor that used a cantilever with a piezo-resistive layer and PDMS. ${ }^{(12,13)}$ The $5 \times 5 \mathrm{~mm}^{2}$ sensor chip was sufficiently small to be attached to a robotic hand. Also reported was a force sensor made from a cantilever with a lead zirconate titanate (PZT) thin film and

*Corresponding author: e-mail: toshihiro-takeshita@aist.go.jp

https://doi.org/10.18494/SAM.2021.2915 
PDMS. ${ }^{(14)}$ The PZT increased the sensitivity and reduced power consumption. In addition, the relationship between PDMS hardness and sensor sensitivity was evaluated.

However, a practical problem of a MEMS cantilever force sensor embedded in an elastomer is breakage during heat treatment. As shown in Fig. 1, after the force sensor is mounted on a package and then mounted on an electronic substrate with other components, solder reflow processing at $250{ }^{\circ} \mathrm{C}$ is performed. The heat causes the elastomer between the cantilever and the package to thermally expand and apply stress to the cantilever. As a result, the base of the MEMS cantilever is broken. The relationship between this breakage and the design of the MEMS cantilever has not been reported.

Here, the relationship between the MEMS cantilever design and the stress caused by thermal expansion of the elastomer was investigated. Specifically, the effect of cantilever thickness and width on the von Mises stress induced by thermal expansion was simulated by finite element methods (FEM) using ANSYS software. Experimental test samples were fabricated from MEMS cantilever chips embedded in PDMS. Fracture tests were then performed to compare the simulated and experimental results.

\section{Materials and Methods}

\subsection{Simulation}

The effect of elastomer thermal expansion on the induced stress of the MEMS cantilever was examined with FEM simulations. Figure 2 shows the simulation model. The cantilever was composed of a 400- $\mu$ m-thick handle Si layer and a device Si layer with thickness $t$. The cantilever had a $1300 \times w \mu \mathrm{m}^{2}$ connection structure and a $1000 \times 1000 \mu \mathrm{m}^{2}$ force-sensing structure. $\mathrm{R}$ structures with a radius of curvature of $200 \mu \mathrm{m}$ were placed at the connection points between the connection and pedestal structures of the cantilever, which was then

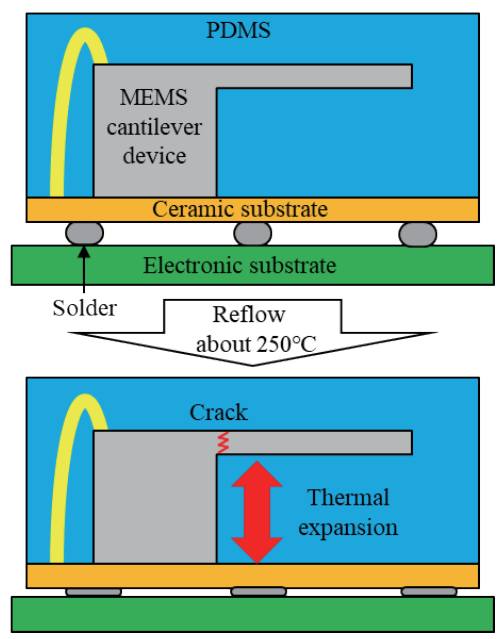

Fig. 1. (Color online) Schematic of breaking of MEMS cantilever device via thermal expansion of PDMS elastomer.

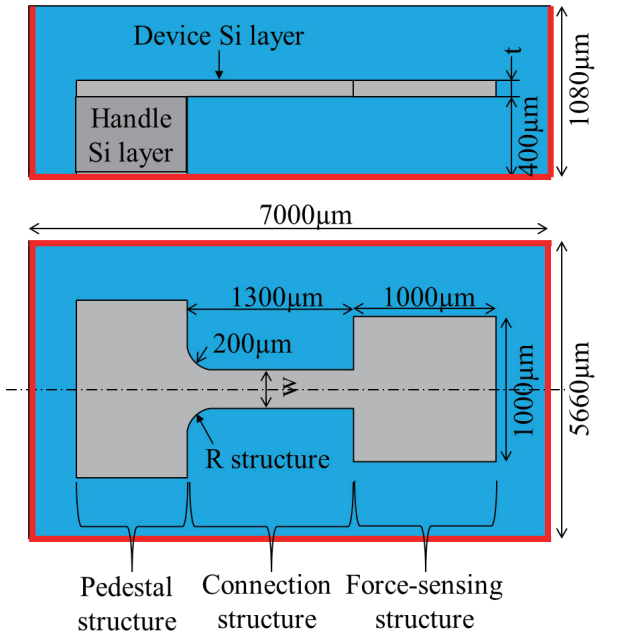

Fig. 2. (Color online) Design of simulation model. 
embedded in elastomer. The size after embedding was $7000 \times 5660 \times 1080 \mu \mathrm{m}^{3}$, which was determined by the experimental ceramic package. The handle Si layer and the active Si layer were single-crystal silicon, and the elastomer was PDMS; their mechanical properties used in this simulation ${ }^{(15-18)}$ are given in Table 1 . The MEMS cantilever and the material properties of PDMS were designed to detect the pushing force of a human fingertip (about $5 \mathrm{~N}$ ). ${ }^{(14)}$

Figure 3(a) shows the cross-sectional view of the simulated deformation and von Mises stress for the $T=10 \mu \mathrm{m}, w=600 \mu \mathrm{m}$ structure at $T=200{ }^{\circ} \mathrm{C}$. This result confirmed that the base of the cantilever connection structure was deformed and swelled upward because the PDMS thermally expanded and deformed. Figure 3(b) shows the simulated MEMS cantilever where the maximum von Mises stress of $7.19 \mathrm{GPa}$ occurred on the edge of the R structure. Figure 4(a) plots the simulated maximum von Mises stress values for thickness $T(5,10 \mu \mathrm{m})$ and width $w(400$, $600 \mu \mathrm{m})$ as a function of temperature $T\left(50,100,150,200\right.$, and $\left.250^{\circ} \mathrm{C}\right)$. The stress increased linearly with temperature, and the maximum von Mises stresses at $T=150{ }^{\circ} \mathrm{C}$ were $5.85 \mathrm{GPa}$ $(t=5 \mu \mathrm{m}, w=600 \mu \mathrm{m}), 5.74 \mathrm{GPa}(t=5 \mu \mathrm{m}, w=400 \mu \mathrm{m}), 4.90 \mathrm{GPa}(t=10 \mu \mathrm{m}, w=600 \mu \mathrm{m})$, and $4.47 \mathrm{GPa}(t=10 \mu \mathrm{m}, w=400 \mu \mathrm{m})$ as shown in Table 2. The von Mises stress maxima were smaller for the narrow $(w=400 \mu \mathrm{m})$ and thicker $(t=10 \mu \mathrm{m})$ cantilevers. Figure $4(\mathrm{~b})$ shows the temperature when the maximum von Mises stress reached $6.2 \mathrm{GPa}$; this is discussed below. Overall, the simulations showed that the thickness and width of the cantilever affected the von Mises stress induced by the PDMS thermal expansion.

Table 1

Mechanical properties of single-crystal silicon and PDMS used in the simulations.

\begin{tabular}{llc}
\hline Materials & Property & Value \\
\hline \multirow{3}{*}{$\mathrm{Si}$} & & $\mathrm{C}_{11} 167.4 \mathrm{GPa}$ \\
& & $\mathrm{C}_{12} 65.2 \mathrm{GPa}$ \\
& & $\mathrm{C}_{14} 79.6 \mathrm{GPa}$ \\
\hline \multirow{3}{*}{ PDMS } & Thermal expansion coefficient & $3.34 \times 10^{-6} /{ }^{\circ} \mathrm{C}$ \\
\cline { 2 - 3 } & Elastic modules & $4.0 \mathrm{MPa}$ \\
\cline { 2 - 3 } & Poisson's ratio & 0.499 \\
\cline { 2 - 3 } & Thermal expansion coefficient & $3.00 \times 10^{-4} /{ }^{\circ} \mathrm{C}$ \\
\hline
\end{tabular}

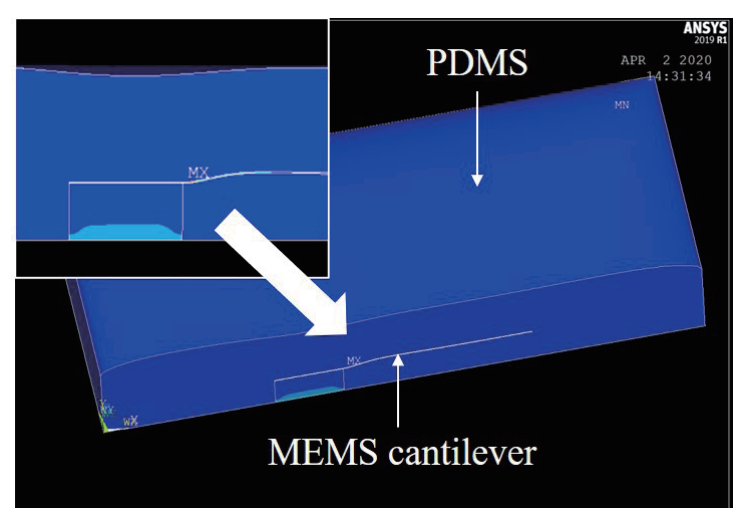

(a)

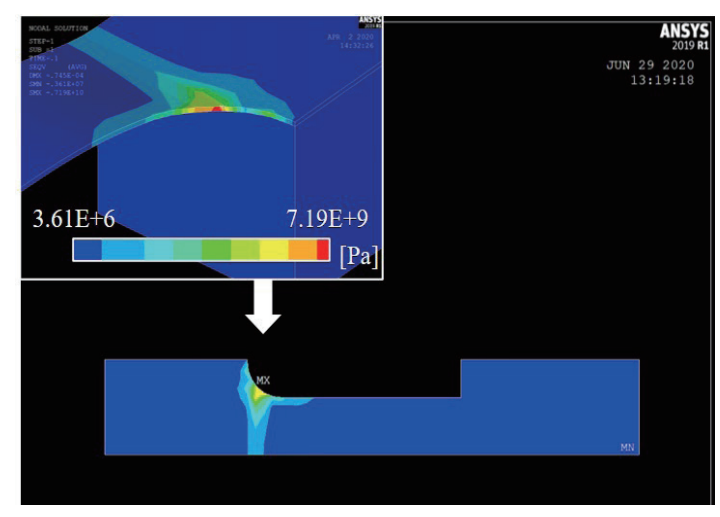

(b)

Fig. 3. (Color online) Cross-sectional simulations of (a) whole model and (b) MEMS cantilever. 


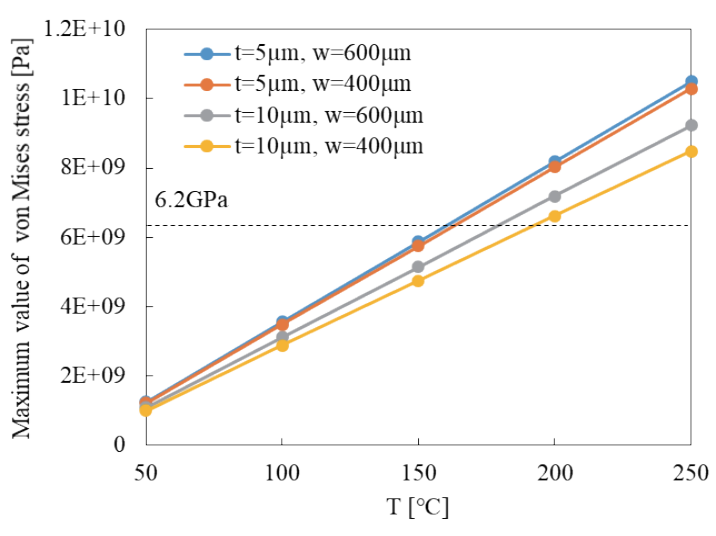

(a)

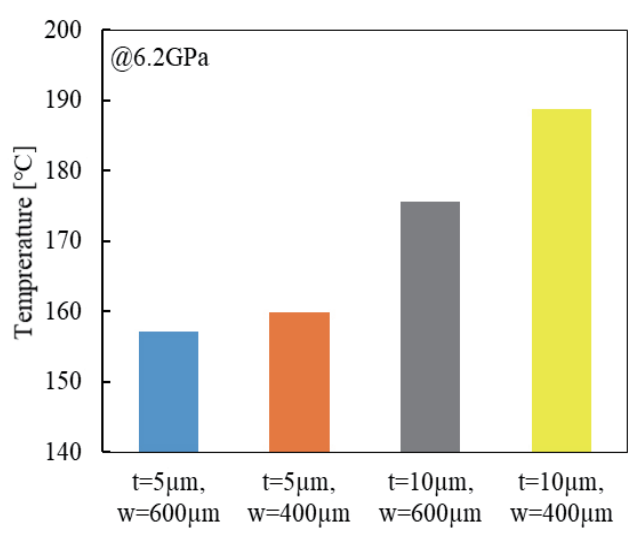

(b)

Fig. 4. (Color online) (a) Simulated maximum von Mises stress vs temperature and (b) temperatures for various structures when the maximum von Mises stress was $6.2 \mathrm{GPa}$.

Table 2

Components of stress tensor and von Mises stress at the point of the maximum von Mises stress $\left(T=150{ }^{\circ} \mathrm{C}\right)$.

\begin{tabular}{lccccccc}
\hline & $\sigma_{x x}(\mathrm{GPa})$ & $\sigma_{y y}(\mathrm{GPa})$ & $\sigma_{z z}(\mathrm{GPa})$ & $\sigma_{x y}(\mathrm{GPa})$ & $\sigma_{y z}(\mathrm{GPa})$ & $\sigma_{x z}(\mathrm{GPa})$ & $\begin{array}{c}\text { von Mises } \\
\text { stress }(\mathrm{GPa})\end{array}$ \\
\hline $\begin{array}{l}t=5 \mu \mathrm{m}, \\
w=600 \mu \mathrm{m}\end{array}$ & 0.71 & 0.20 & 0.06 & -0.66 & -1.54 & 2.91 & 5.85 \\
\hline $\begin{array}{l}t=5 \mu \mathrm{m}, \\
w=400 \mu \mathrm{m}\end{array}$ & 0.75 & 0.21 & 0.05 & -0.52 & -1.61 & 2.82 & 5.74 \\
\hline $\begin{array}{l}t=10 \mu \mathrm{m}, \\
w=600 \mu \mathrm{m}\end{array}$ & 0.40 & 0.11 & 0.01 & -0.28 & -0.68 & 2.73 & 4.90 \\
\hline $\begin{array}{l}t=10 \mu \mathrm{m}, \\
w=400 \mu \mathrm{m}\end{array}$ & 0.36 & 0.10 & 0.01 & -0.25 & -0.61 & 2.49 & 4.47 \\
\hline
\end{tabular}

\subsection{Experimental results}

MEMS cantilever chips were fabricated to perform breaking tests. As shown in Fig. 5, the fabrication was as follows. ${ }^{(1)}$ A 200 -nm-thick Pt/Ti wiring film was sputter deposited on a silicon-on-insulator substrate (device Si layer thickness: 5, $10 \mu \mathrm{m}$ ). The Pt/Ti layer was used for breakage detection and was etched (2) via ion milling. ${ }^{(3)}$ The device Si layer was subjected to reactive ion etching (RIE). ${ }^{(4)}$ Etching of the handle Si layer was performed via deep RIE, followed by dry etching of the $\mathrm{SiO}_{2}$ layer. ${ }^{(5)}$ The MEMS cantilever was mounted on a ceramic package with an adhesive paste applied to the bottom of the pedestal structure. ${ }^{(6)} \mathrm{Au}$ wire bonding connected the ceramic package to the $\mathrm{Pt} / \mathrm{Ti}$ layer of the cantilever. ${ }^{(7)}$ Finally, the MEMS cantilever chip was embedded in PDMS (KE-106, Shin-Etsu Chemical Co., Ltd., Japan) and cured at $100{ }^{\circ} \mathrm{C}$ for $1 \mathrm{~h}$. The ratio of the PDMS base and curing agents was 10:1.

Figure 6(a) is a photograph of a test sample where a MEMS cantilever is fixed in the center of a ceramic package. The $\mathrm{Pt} / \mathrm{Ti}$ wiring film was formed on the surface of the connection and force-sensing structure, and two electrode pads were formed on the surface of the pedestal structure. 


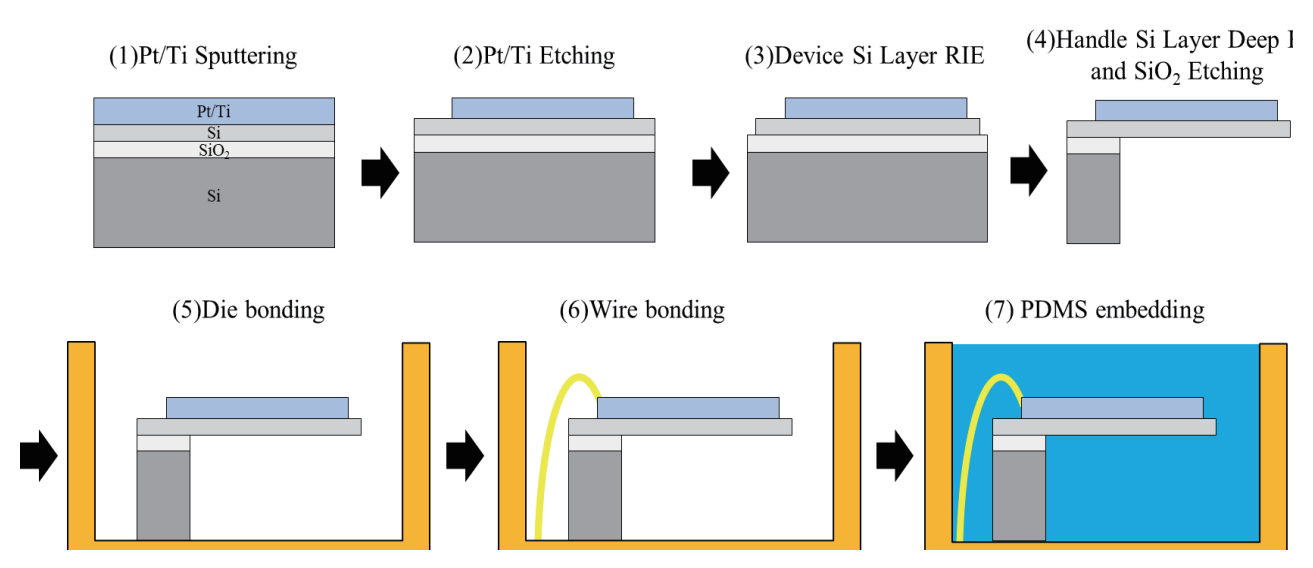

Fig. 5. (Color online) Fabrication of MEMS cantilever and embedded test sample.

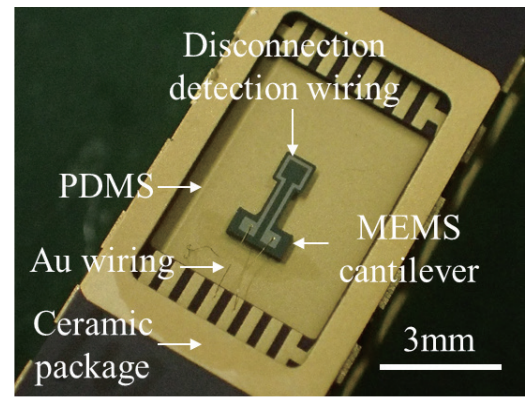

(a)

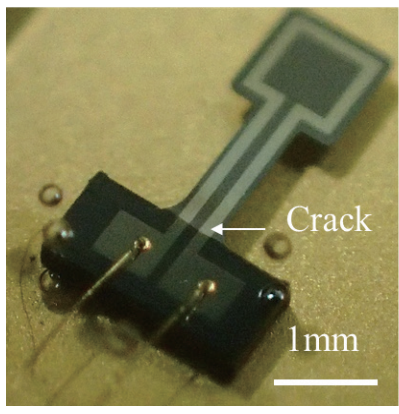

(b)

Fig. 6. (Color online) Photographs of test sample (a) before and (b) after experiment.

These electrode pads and those on the ceramic package were connected by Au wire. Eight test samples with thicknesses $T=5 \mu \mathrm{m}$ and $T=10 \mu \mathrm{m}$ and widths $w=400 \mu \mathrm{m}$ and $w=600 \mu \mathrm{m}$ were prepared and heated. After $5 \mathrm{~min}$ at temperature $T$, the resistance of the $\mathrm{Pt} / \mathrm{Ti}$ breakage detection wiring was measured. Once the cantilever broke, the resistance increased sharply, and the $T$ was recorded. Figure 6(b) is a photograph of a MEMS cantilever chip after breaking. The fracture occurred at the base of the connection structure, as predicted by the simulations.

Figure 7(a) is a schematic of the experimental setup and Fig. 7(b) plots the average breaking temperature and standard deviation for the eight test samples. A higher breaking temperature indicated higher thermal stability. For the $T=5 \mu \mathrm{m}$ samples, there was no significant difference between the $w=600 \mu \mathrm{m}\left(166.3{ }^{\circ} \mathrm{C}\right)$ and $w=400 \mu \mathrm{m}\left(167.9^{\circ} \mathrm{C}\right)$ samples, whereas the $T=10 \mu \mathrm{m}$, $w=400 \mu \mathrm{m}$ test sample had a higher breaking temperature $\left(188.3{ }^{\circ} \mathrm{C}\right)$ than did the $t=10 \mu \mathrm{m}$, $w=600 \mu \mathrm{m}$ sample $\left(174.1^{\circ} \mathrm{C}\right)$. Also, both the $w=600 \mu \mathrm{m}$ and $w=400 \mu \mathrm{m}$ samples with $T=10 \mu \mathrm{m}$ had higher breaking temperatures than did the $T=5 \mu \mathrm{m}$ samples. Hence, the thicker cantilevers had higher thermal stabilities. 


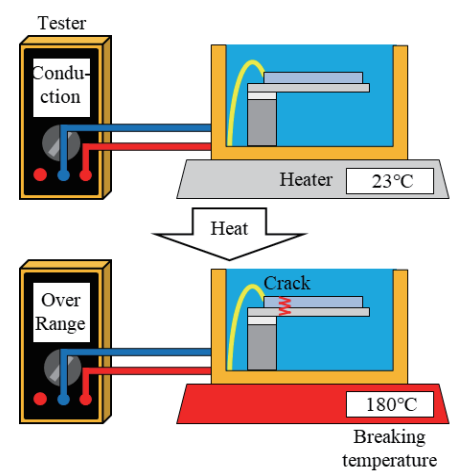

(a)

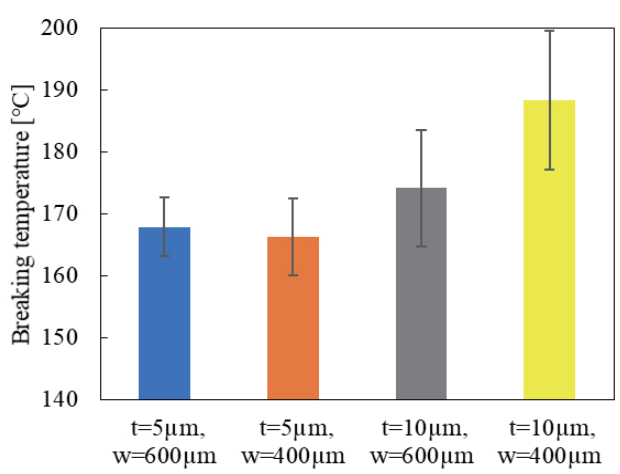

(b)

Fig. 7. (Color online) Schematic of (a) experimental setup and (b) results.

\section{Discussion}

The simulations indicated that thicker and narrower cantilevers reduced the von Mises stress. Thicker cantilevers had a larger area moment of inertia; specifically, the area moment of inertia for the $t=10 \mu \mathrm{m}$ cantilever was eight times that of the $T=5 \mu \mathrm{m}$ cantilever. Therefore, the thicker cantilevers were not greatly deformed when the PDMS expanded. Meanwhile, narrower widths reduced the stress-receiving area. Because the deformation of the cantilever was induced by the PDMS thermal expansion, the stress on the cantilever was caused by the displacement (not pressure) of the entire area of its back surface. Figure 8 shows (a) an image of the deformed cantilever ( $z$-direction, $T=150{ }^{\circ} \mathrm{C}$ ) and (b) the displacement of the cantilever root $(100 \mu \mathrm{m}$ from the pedestal structure, $\mathrm{A}-\mathrm{A}^{\prime}$ cross-section). The deformation of the cantilever is mountainshaped, and the displacement at $w=600 \mu \mathrm{m}$ is larger than the displacement at $w=400 \mu \mathrm{m}$. The reason for the mountain shape is that the ceramic package holds four sides of the PDMS, and the central part of the ceramic package $(y=0)$ rises. The shape depends on the cantilever width as shown in Fig. 8(b). Therefore, the wider cantilever $(w=600 \mu \mathrm{m})$ is displaced largely in the $z$-direction. This can be confirmed by the different $\sigma_{x y}$ and $\sigma_{x z}$ values between the cantilever $(t=5 \mu \mathrm{m}, w=600 \mu \mathrm{m})$ and the cantilever $(t=5 \mu \mathrm{m}, w=400 \mu \mathrm{m})$ as shown in Table 2. Also, the nonuniform deformation causes torsional stress around the $x$-axis. Because the torsional stress is dependent on the distance from the center of the cantilever $(y=0)$ to the R structure $(y=w / 2)$, larger stress is applied to the wider cantilever. This can also be confirmed by the different $\sigma_{y z}$ values between the cantilever $(t=5 \mu \mathrm{m}, w=600 \mu \mathrm{m})$ and the cantilever $(t=5 \mu \mathrm{m}, w=400 \mu \mathrm{m})$ as shown in Table 2.

The experimental results for cantilevers with $T=5 \mu \mathrm{m}$ and $w=600 \mu \mathrm{m}$ indicated higher thermal stabilities than those with $w=400 \mu \mathrm{m}$, which did not agree with the simulations. However, the difference between the average breaking temperatures was only $1.6{ }^{\circ} \mathrm{C}$, which was within the standard deviation. Moreover, the effect of the width was small in the simulations for $T=5 \mu \mathrm{m}$. Therefore, this disagreement could be resolved with more experiments. When $T=10 \mu \mathrm{m}$ and $w=400 \mu \mathrm{m}$, the breaking temperature was $188.3{ }^{\circ} \mathrm{C}$, which, when compared 


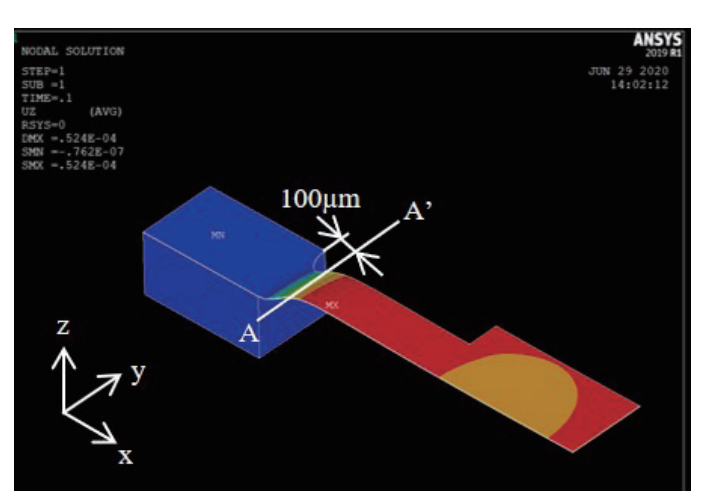

(a)

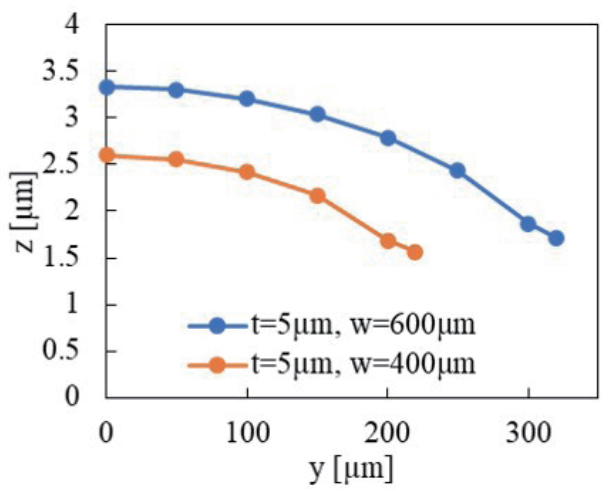

(b)

Fig. 8. (Color online) Image of (a) the deformed cantilever and (b) displacement at the root of the cantilever (A-A' cross section).

with the simulations [Fig. 4(b)], indicated a von Mises stress of $6.2 \mathrm{GPa}$. The theoretical breaking strength of single-crystal silicon is $30 \mathrm{GPa}^{(19)}$ Hence, the breakage of the singlecrystal silicon occurred via a von Mises stress that was much smaller than the theoretical value because of defects that formed during the MEMS process. Surface defects reduce the breaking strength, ${ }^{(20)}$ and, in particular, because the von Mises stress was concentrated on the R structures, the effects of surface defects cannot be ignored. Therefore, the breaking strength of the cantilever was less than the theoretical value.

Overall, the results indicated the following findings.

(1) The design of the cantilever affects the thermal stability. Thicker and narrower cantilevers had higher thermal stabilities.

(2) Stress was concentrated on the R structures.

(3) The simulated and experimental results agreed for the most part. Increasing the number of test samples could improve the agreement.

(4) To increase the breaking strength, it is important to reduce the number of surface defects formed in the MEMS process.

This work had limitations in the simulations, experiments, and practical applications. In the simulations, the thermal expansion coefficient and Young's modulus for the single-crystal silicon and PDMS were constant. However, both are temperature-dependent. Thus, we could improve the simulation accuracy by using more accurate mechanical properties (including thermal dependence) of the materials. In the experiments, the standard deviations were not small because the breaking strength was greatly affected by the surface quality. Thus, the number of samples should be increased. Finally, when used as a force sensor, the thickness of a MEMS cantilever embedded in an elastomer greatly affects the force sensitivity. That is, the sensitivity increases with decreasing thickness. Therefore, cantilever design requires consideration of both thermal endurance and sensitivity. 


\section{Conclusions}

The relationship between the thermal expansion of the elastomer and the breaking temperature of an embedded MEMS cantilever was characterized. In particular, the effects of cantilever thickness and width on the thermal stability were determined. Simulation and experimental results both indicated that thicker and narrower cantilevers had higher thermal stabilities. In the future, the effects of the thermal expansion coefficient and Young's modulus of the elastomer on thermal stability will be investigated. In addition, our goal is to design and fabricate a MEMS cantilever force sensor with a thermal stability above $250{ }^{\circ} \mathrm{C}$.

\section{References}

1 T. Takeshita, T. Iwasaki, K. Harisaki, H. Ando, E. Higurashi, and R. Sawada: Sens. Mater. 26 (2014) 547. https://doi.org/10.18494/SAM.2014.1014

2 T. Takeshita, Y. Peng, N. Morita, H. Ando, E. Higurashi and R. Sawada: Proc. 14th Joint Int. IMEKO TC1 + TC7 + TC 13 Symp. (IMEKO 2011) 133. https://www.db-thueringen.de/receive/dbt mods 00019462

3 T. Takeshita, T. Iwasaki, E. Higurashi, and R. Sawada: Sens. Mater. 25 (2013) 609. https://doi.org/10.18494/ SAM.2013.924

4 R. Sawada, E. Higurashi, T. Itoh, and I. Ishikawa: Sens. Actuators, A 136 (2007) 161. https://doi.org/10.1016/ j.sna.2006.10.055

5 Y. Qin, Y. Zhao, Y. Li, Y. Zhao, and P. Wang: Sensors 16 (2016) 513. https://doi.org/10.3390/s16040513

6 T. Iwasaki, T. Takeshita, Y. Arinaga, and R. Sawada: Sens. Mater. 25 (2013) 601. https://doi.org/10.18494/ SAM.2013.923

7 N. T. Vinh, N. B. Khiem, H. Takahashi, K. Matsumoto, and I. Shimoyama: Sens. Actuators, A 215 (2014) 167. https://doi.org/10.1016/j.sna.2013.09.002

8 T. Takeshita, K. Harisaki, H. Ando, E. Higurashi, H. Nogami, and R. Sawada: Precis. Eng. 45 (2016) 136. https://doi.org/10.1016/j.precisioneng.2016.02.004

9 M. Ohka, Y. Mitsuya, I. Higashioka, and H.Kabeshita: Robotica 23 (2005) 457. https://doi.org/10.1017/ $\underline{\mathrm{S} 0263574704001535}$

10 K. Noda, H. Onoe, E. Iwase, K. Matsumoto, and I. Shimoyama: J. Micromech. Microeng. 22 (2012) 115025-1. https://doi.org/10.1088/0960-1317/22/11/115025

11 K. Noda, K. Hoshino, K. Matsumoto, and I. Shimoyama: Sens. Actuators, A 127 (2006) 295. https://doi. org/10.1016/j.sna.2005.09.023

12 M. Sohgawa, A. Nozawa, H. Yokoyamay, and T. Kanashimay: Proc. IEEE SENSORS 2014 (IEEE 2014$) 1$. https://doi.org/10.1109/ICSENS.2014.6985362

13 R. Araki, T. Abe, H. Noma, and M. Sohgawa: IEEJ T. Sensor. Micromach. 137 (2017) 212. https://doi. org/10.1541/ieejsmas

14 T.Takeshita, T. Kobayashi, R.Takei, T. Itoh, and S. Takamatsu: Jpn. J. Appl. Phys. 56 (2017) 04CC04-1. https:// doi.org/10.7567/JJAP.56.04CC04

15 D. Fuard, T. T. Chevolleau, S. Decossas, P. Tracqui, and P. Schiavone: Microelectron. Eng. 85 (2008) 1289. https://doi.org/10.1016/j.mee.2008.02.004

16 S. Dogru, B. Aksoy, H. Bayraktar, and B. E. Alace: Polym. Test. 69 (2018) 375. https://doi.org/10.1016/ j.polymertesting.2018.05.044

17 C. Jin, Z. Wang, A. A. Volinsky, A. Sharfeddin, and N. D. Gallant: Polym. Test. 56 (2016) 329. https://doi. org/10.1016/j.polymertesting.2016.10.034

18 M. Levinshtein, S. Rumyantsev, and M. Shur: Handbook Series on Semiconductor Parameters 1 (1996) 1. https://doi.org/10.1142/2046-vol1

19 E. Orowan: Rep. Prog. Phys. 12 (1949) 185. https://iopscience.iop.org/article/10.1088/0034-4885/12/1/309

20 A. Uesugi, Y. Hirai, K. Sugano, T. Tsuchiya, and O. Tabata: T. JSME. A 79 (2013) 1191. https://doi.org/10.1299/ kikaia.79.1191 